\title{
Connections between TMD and Collinear Twist-3 Functions within the Context of Single-Spin Asymmetries
}

Daniel PITONYAK*

Penn State University Berks

E-mail: dap67@psu.edu

Leonard GAMBERG

Penn State University Berks

E-mail: lpg10@psu.edu

Andreas METZ

Temple University

E-mail: metza@temple.edu

\section{Alexei PROKUDIN}

Penn State University Berks

Theory Center, Jefferson Lab

E-mail: avp5627@psu.edu

\begin{abstract}
We review the theoretical framework underlying the description of transverse single-spin asymmetries (SSAs) for both collinear and transverse-momentum-dependent (TMD) processes. For the latter, we discuss modifications to the widely used CSS formalism that are needed to address certain issues that have arisen in TMD analyses. After these improvements, one can derive rigorous relations between collinear and TMD functions. Moreover, we show how both collinear and TMD SSA observables are driven by multi-parton correlations, which allows for a future global extraction to be performed including data from both types of measurements.
\end{abstract}

QCD Evolution 2017 -QCDEV2017-

22-26 MAY 2017

Jefferson Lab (JLAB), Newport News Virginia, USA

\footnotetext{
* Speaker.
} 


\section{Introduction}

In the last 40 year, transverse single-spin asymmetries (SSAs) have arisen in a variety of reactions. Some of these are collinear observables, like $p^{\uparrow} p \rightarrow\{h, \gamma$, jet $\} X[1,2,3,4,5,7,8,9,6,10,11,12$, 13], $p p \rightarrow h^{\uparrow} X$ [14], $\ell N^{\uparrow} \rightarrow h X[15,16], \ell N^{\uparrow} \rightarrow \ell^{\prime} X$ [17, 18], and $e^{+} e^{-} \rightarrow h^{\uparrow} X$ [19]; and others are transverse-momentum-dependent (TMD) processes, like $\ell N \rightarrow \ell^{\prime} h X$ [20, 22, 23, 24], $h_{1} h_{2} \rightarrow$ $\left\{\ell \bar{\ell}, W^{ \pm}, Z\right\} X[25,26]$, and $e^{+} e^{-} \rightarrow h_{1} h_{2} X[19,27,28,29,30]$. The former are studied within collinear twist-3 factorization [31, 32, 33, 34, 35, 36, 37, 38, 39, 40, 41, 42, 43] (since there is only one large scale $P_{T} \gg \Lambda_{Q C D}$ ) while the latter are analyzed within TMD factorization (since there are two scales $\Lambda_{Q C D} \sim P_{T} \ll Q$ ) using either the generalized parton model approach [44, 45, 46, $47,48,49]$ or the Collins-Soper-Sterman (CSS) formalism [50, 51, 52, 53, 54, 55]. Both collinear and TMD SSAs provide valuable insight into the internal structure of hadrons, including quarkgluon correlations and spin-orbit effects. In Sec. 2 we review the twist-3 formalism and its current description of collinear SSAs, while in Sec. 3 we do the same for TMD SSAs, focusing on the CSS formalism. Moreover, we discuss how both collinear and TMD SSAs are driven by multi-parton correlations, which ultimately will allow for a global analysis of both types of measurements. We also briefly discuss some recent modifications to the original CSS framework. Because of these improvements, we can derive rigorous relations between collinear twist-3 and TMD functions, which we do in Sec. 4. We conclude in Sec. 5.

\section{SSAs within Collinear Factorization}

Here we will focus on single-inclusive reactions where an unpolarized proton collides with a transversely polarized one. We refer the reader to Ref. [56] for a more comprehensive review of this topic. In the collinear twist- 3 approach, one writes the differential cross section for $p^{\uparrow} p \rightarrow C X$ as

$$
\begin{aligned}
d \sigma\left(S_{T}\right) & =H \otimes f_{a / p^{\uparrow}(3)} \otimes f_{b / p(2)} \otimes D_{C / c(2)} \\
& +H^{\prime} \otimes f_{a / p^{\dagger}(2)} \otimes f_{b / p(3)} \otimes D_{C / c(2)} \\
& +H^{\prime \prime} \otimes f_{a / p^{\dagger}(2)} \otimes f_{b / p(2)} \otimes D_{C / c(3)} .
\end{aligned}
$$

In Eq. (2.1), $f_{a / p^{\uparrow}(t)}$ is the parton distribution function (PDF) associated with parton $a$ in the transversely polarized proton $p^{\uparrow}$ (and similarly for $f_{b / p(t)}$ ), while $D_{C / c(t)}$ is the fragmentation function (FF) associated with hadron $C$ in parton $c$. The twist of the functions is indicated by $t$. The factors $H, H^{\prime}$, and $H^{\prime \prime}$ are the hard parts for each term, and the symbol $\otimes$ denotes convolutions in the appropriate momentum fractions. The hard scale for the reaction is set by the transverse momentum of the outgoing particle, $P_{C T} \gg \Lambda_{Q C D}$, and $S_{T}$ is the transverse spin vector of $p^{\uparrow}$.

We would like to extract the twist-3 functions in Eq. (2.1) because, as we will see, these functions give us access to multi-parton correlations in hadrons. However, since the individual terms in (2.1) add together and cannot be "projected out", one must try to isolate certain pieces through experiments that are only mainly sensitive to a specific function or functions. For example, if one looks at direct photon production, which eliminates the third term in (2.1) since there is no fragmentation, we find such a process is dominated by a quark-gluon-quark PDF, the Qiu-Sterman (QS) function $F_{F T}(x, x)[59,60]$. The QS function has an important, model-independent relation to the TMD Sivers function [57] $f_{1 T}^{\perp}\left(x, k_{T}^{2}\right)$ that enters SSAs in processes like semi-inclusive 
deep-inelastic scattering (SIDIS) and Drell-Yan (DY), which we will cover in Sec. 3. The identity reads [58]

$$
\pi F_{F T}(x, x)=\left.\left.\int d^{2} k_{\mathrm{T}} \frac{k_{T}^{2}}{2 M_{P}^{2}} f_{1 T}^{\perp}\left(x, k_{T}\right)\right|_{S I D I S} \equiv f_{1 T}^{\perp(1)}(x)\right|_{S I D I S}=-\left.f_{1 T}^{\perp(1)}(x)\right|_{D Y} .
$$

We note that this relation is derived using the naïve definitions of the operators for these functions (without any soft-gluon radiation effects), and we will see in Sec. 4 how to approximately recover this form within "full QCD" via the CSS formalism.

Moreover, a main experimental focus has been on the case where $C=\pi$, which was initially thought to be caused by the QS function [36, 61]. However, several works in the literature showed later that this cannot be the case $[62,63,64]$. What is currently understood as the cause of SSAs in pion production is the third term in (2.1) involving twist-3 fragmentation effects [65, 66]. The functions we are sensitive to from this mechanism are $\tilde{H}(z)$ and $H_{1}^{\perp(1)}(z)$ [41, 67]. The former is an integrated twist-3 TMD FF, while the latter in the first-moment of the Collins function,

$$
H_{1}^{\perp(1)}(z)=z^{2} \int d^{2} p_{\perp} \frac{\vec{p}_{\perp}^{2}}{2 M_{h}^{2}} H_{1}^{\perp}\left(z, z^{2} p_{\perp}^{2}\right) .
$$

Again we will see in Sec. 4 how to approximately recover this relation in the CSS formalism. Both $\tilde{H}\left(z, z^{2} p_{\perp}^{2}\right)$ and $H_{1}^{\perp}\left(z, z^{2} p_{\perp}^{2}\right)$ enter asymmetries in SIDIS and $e^{+} e^{-} \rightarrow h_{1} h_{2} X[68,69]$. We emphasize that both $\tilde{H}(z)$ and $H_{1}^{\perp(1)}(z)$ can be written as integrals of a quark-gluon-quark FF $\hat{H}_{F U}^{\mathfrak{S}}\left(z, z_{1}\right)$ by using QCD equation of motion relations (EOMRs) and so-called Lorentz invariance relations (LIRs) [67]. That is, multi-parton correlators are what underlie SSAs in collinear observables. Since $\tilde{H}(z)$ and $H_{1}^{\perp(1)}(z)$ couple to transversity $h_{1}(x)$ at larger $x$ values, where the function is currently unconstrained, we can extract $h_{1}(x)$ in this region through SSAs in pion production $[65,66]$.

\section{SSAs within TMD Factorization}

Here we will focus on the Sivers and Collins TMD functions that lead to different azimuthal modulations in SIDIS, Drell-Yan, and $e^{+} e^{-} \rightarrow h_{1} h_{2} X$. Within the CSS formalism, these functions can be written in " $b$-space" as (at leading-order) $[52,53,54,55]$

$$
\begin{aligned}
& \tilde{f}_{1 T}^{\perp(1)}\left(x, b_{T} ; Q^{2}, \mu_{Q}\right) \sim F_{F T}\left(x, x ; \mu_{b_{*}}\right) \exp \left[-S_{\text {pert }}\left(b_{*}\left(b_{T}\right) ; \mu_{b_{*}}, Q, \mu_{Q}\right)-S_{N P}^{f_{1 T}^{\perp}}\left(b_{T}, Q\right)\right], \\
& \tilde{H}_{1}^{\perp(1)}\left(z, b_{T} ; Q^{2}, \mu_{Q}\right) \sim H_{1}^{\perp(1)}\left(z ; \mu_{b_{*}}\right) \exp \left[-S_{p e r t}\left(b_{*}\left(b_{T}\right) ; \mu_{b_{*}}, Q, \mu_{Q}\right)-S_{N P}^{H_{1}^{\perp}}\left(b_{T}, Q\right)\right],
\end{aligned}
$$

where

$$
b_{*}\left(b_{T}\right) \equiv \sqrt{\frac{b_{T}^{2}}{1+b_{T}^{2} / b_{\max }^{2}}}, \quad \mu_{b_{*}} \equiv \frac{C_{1}}{b_{*}\left(b_{T}\right)},
$$

The quantities $S_{\text {pert }}$ and $S_{N P}$ are the perturbative and non-perturbative Sudakov factors, where former is universal and the latter is different for each TMD [52, 53, 54, 55]. The collinear twist-3 functions $F_{F T}\left(x, x ; \mu_{b_{*}}\right)$ and $H_{1}^{\perp(1)}\left(z ; \mu_{b_{*}}\right)$ arise from the Operator Product Expansion (OPE) at small $b_{T}$. The functions $\left[b_{T} \tilde{f}_{1 T}^{\perp(1)}\left(x, b_{T} ; Q^{2}, \mu_{Q}\right)\right]$ and $\left[b_{T} \tilde{H}_{1}^{\perp(1)}\left(z, b_{T} ; Q^{2}, \mu_{Q}\right)\right]$ are proportional to 
the derivative of the Fourier transforms (FT) of the TMD Sivers and Collins functions, respectively $[53,70]$. The FT-TMDs have two scale arguments: $\mu$, which is the renormalization scale, and $\zeta$, which parameterizes how the effects of soft-gluon radiation are partitioned between the FTTMDs, where $\zeta_{A} \zeta_{B}=Q^{4}$. We use the freedom from the renormalization group to set $\zeta_{A}=\zeta_{B}=Q^{2}$ and $\mu=C_{2} Q \equiv \mu_{Q}$. The constant $C_{2}$ is chosen to optimize the accuracy of the perturbation theory for the hard scattering coefficients.

We see immediately from Eqs. (3.1), (3.2) that the TMDs and their associated observables are also driven by multi-parton correlators just like the collinear SSAs discussed in Sec. 2. That is, extractions of TMD functions from the aforementioned observables actually fit collinear twist- 3 functions (along with the non-perturbative Sudakov factor) [54, 55]. Therefore, one can include collinear SSA data along with data on the Sivers and Collins asymmetries in a global analysis in order to obtain information on $F_{F T}(x, x), \hat{H}_{F U}^{\mathfrak{S}}\left(z, z_{1}\right)$ (via $\tilde{H}(z)$ and $H_{1}^{\perp(1)}(z)$ ), and $h_{1}(x)$. In general, one can say that all transverse spin effects are caused by multi-parton correlations in hadrons.

\section{Relations between Collinear and TMD Functions}

In Eqs. (2.2), (2.3) we discussed two important relations between TMD and collinear twist-3 functions that result from their naïve operator definitions. These identities have not only been routinely used in phenomenology (see, e.g., Refs. [59, 60, 63, 64, 65]), but also give important physical interpretations of these functions as being connected to average parton transverse momentum [71]. However, these important connections actually break down within the original CSS formalism discussed in Sec. 3. For example, one finds [72, 73]

$$
\int d^{2} k_{\mathrm{T}} \frac{k_{T}^{2}}{2 M_{P}^{2}} f_{1 T}^{\perp}\left(x, k_{T} ; Q^{2}, \mu_{Q}\right)=\tilde{f}_{1 T}^{\perp(1)}\left(x, b_{T} \rightarrow 0 ; Q^{2}, \mu_{Q}\right)=0+O\left(\alpha_{s}(Q)\right) .
$$

The source of this behavior is that $b_{*}\left(b_{T} \rightarrow 0\right)=0$ so that $\mu_{b_{*}} \rightarrow \infty$ in this limit, which leads to a large logarithm in the perturbative Sudakov factor. To cure this issue, the authors of Ref. [72] suggested to modify the so-called $W$-term of the $b$-space cross section by $b_{T} \rightarrow b_{c}\left(b_{T}\right)$, where

$$
b_{c}\left(b_{T}\right)=\sqrt{b_{T}^{2}+\left(\frac{b_{0}}{C_{5} Q}\right)^{2}}=\sqrt{b_{T}^{2}+b_{\text {min }}^{\prime 2}},
$$

with $b_{0} \equiv 2 \exp \left(-\gamma_{E}\right), C_{5}$ a constant, and $b_{\min }^{\prime} \equiv b_{0} /\left(C_{5} Q\right)$, which cuts $b_{T}$ off at $O(1 / Q)$. However, for polarized observables (like the Sivers and Collins effects) this modification should be implemented by only replacing $b_{T}$ with $b_{c}\left(b_{T}\right)$ in the scalar functions that undergo CSS evolution and not to any kinematic, $b_{T}$-dependent prefactors [73].

For example, in

$$
\tilde{W}\left(b_{\mathrm{T}}, Q, S\right)=\tilde{W}_{\mathrm{UU}}\left(b_{T}, Q\right)-i M_{P} \varepsilon^{i j} b_{T}^{i} S_{T}^{j} \tilde{W}_{\mathrm{UT}}^{\mathrm{Sivers}}\left(b_{T}, Q\right)+\ldots,
$$

the $b_{T} \rightarrow b_{c}\left(b_{T}\right)$ replacement only takes place in $\tilde{W}_{\mathrm{UU}}\left(b_{T}, Q\right)$ and $\tilde{W}_{\mathrm{UT}}^{\text {Sivers }}\left(b_{T}, Q\right)$ (the ellipsis is for other azimuthal modulations, like the Collins effect), and not, e.g., in the $b_{T}^{i}$ of the prefactor of the 
second term [73]. This implies the functions in (3.1), (3.2) become [73]

$$
\begin{aligned}
& \tilde{f}_{1 T}^{\perp(1)}\left(x, b_{c}\left(b_{T}\right) ; Q^{2}, \mu_{Q}\right) \sim F_{F T}(x, x ; \bar{\mu}) \exp \left[-S_{p e r t}\left(b_{*}\left(b_{c}\left(b_{T}\right)\right) ; \bar{\mu}, Q, \mu_{Q}\right)-S_{N P}^{f_{1 T}^{\perp}}\left(b_{c}\left(b_{T}\right), Q\right)\right], \\
& \tilde{H}_{1}^{\perp(1)}\left(z, b_{c}\left(b_{T}\right) ; Q^{2}, \mu_{Q}\right) \sim H_{1}^{\perp(1)}(z ; \bar{\mu}) \exp \left[-S_{p e r t}\left(b_{*}\left(b_{c}\left(b_{T}\right)\right) ; \bar{\mu}, Q, \mu_{Q}\right)-S_{N P}^{H_{\perp}^{\perp}}\left(b_{c}\left(b_{T}\right), Q\right)\right],
\end{aligned}
$$

where

$$
b_{*}\left(b_{c}\left(b_{T}\right)\right)=\sqrt{\frac{b_{T}^{2}+b_{\min }^{\prime 2}}{1+b_{T}^{2} / b_{\max }^{2}+b_{\min }^{\prime 2} / b_{\max }^{2}}}, \quad \bar{\mu} \equiv \frac{C_{1}}{b_{*}\left(b_{c}\left(b_{T}\right)\right)},
$$

with $b_{\min }^{\prime}$ defined after (4.2). With these modifications, we now find [73]

$$
\begin{aligned}
\int d^{2} k_{T} \frac{k_{T}^{2}}{2 M_{P}^{2}} f_{1 T}^{\perp}\left(x, k_{T} ; Q^{2}, \mu_{Q}\right) & =\tilde{f}_{1 T}^{\perp(1)}\left(x, b_{\text {min }}^{\prime} ; Q^{2}, \mu_{Q}\right) \\
& =\pi F_{F T}\left(x, x ; \mu_{c}\right)+O\left(\alpha_{s}(Q)\right)+O\left((m / Q)^{p}\right) \\
z^{2} \int d^{2} p_{\perp} \frac{p_{\perp}^{2}}{2 M_{h}^{2}} H_{1}^{\perp}\left(z, z^{2} p_{\perp}^{2} ; Q^{2}, \mu_{Q}\right) & =\tilde{H}_{1}^{\perp(1)}\left(z, b_{\text {min }}^{\prime} ; Q^{2}, \mu_{Q}\right) \\
& =H_{1}^{\perp(1)}\left(z ; \mu_{c}\right)+O\left(\alpha_{s}(Q)\right)+O\left((m / Q)^{p}\right)
\end{aligned}
$$

where $\mu_{c} \equiv \lim _{b_{T} \rightarrow 0} \bar{\mu}$ with $\bar{\mu}$ given in (4.6). We see that the parton model (or naïve operator definition) interpretation of TMDs is approximately restored (i.e., to leading order).

\section{Summary and Outlook}

We have reviewed the current theoretical status of transverse SSAs in both collinear and TMD observables. Collinear SSAs are described using collinear twist-3 functions, while the current formulation of TMD evolution through the CSS formalism also introduces collinear twist-3 functions through the OPE. Therefore, experimental data from both collinear and TMD SSAs can be used in a global analysis of both observables. Moreover, the combination of EOMRs and LIRs allows all twist-3 functions to be written as multi-parton correlators. These multi-parton correlations, therefore, are what drive all SSAs. We have also discussed how improvements to the original CSS formalism allow one (at leading-order) to rederive the naïve operator relations between TMD and collinear functions, which, thus, approximately restore their parton-model physical interpretation.

\section{Acknowledgments}

This material is based upon work supported by the U.S. Department of Energy, Office of Science, Office of Nuclear Physics under Award No. DE-FG02-07ER41460 (L.G.), No. DE-AC0506OR23177 (A.P.), by the National Science Foundation under Contract No. PHY-1516088 (A.M.), No. PHY-1623454 (A.P.), and within the framework of the TMD Topical Collaboration. 


\section{References}

[1] R. D. Klem et al. Phys. Rev. Lett. 36, 929 (1976).

[2] D. L. Adams et al. [E581 and E704 Collaborations], Phys. Lett. B 261, 201 (1991); D. L. Adams et al. [E704 Collaboration], Phys. Lett. B 264, 462 (1991).

[3] K. Krueger, C. Allgower, T. Kasprzyk, H. Spinka, D. Underwood, A. Yokosawa, G. Bunce and H. Huang et al., Phys. Lett. B 459, 412 (1999).

[4] C. E. Allgower, K. W. Krueger, T. E. Kasprzyk, H. M. Spinka, D. G. Underwood, A. Yokosawa, G. Bunce and H. Huang et al., Phys. Rev. D 65, 092008 (2002).

[5] J. Adams et al. [STAR Collaboration], Phys. Rev. Lett. 92, 171801 (2004) [hep-ex/0310058].

[6] B. I. Abelev et al. [STAR Collaboration], Phys. Rev. Lett. 101, 222001 (2008) [arXiv:0801.2990 [hep-ex]].

[7] S. S. Adler et al. [PHENIX Collaboration], Phys. Rev. Lett. 95, 202001 (2005) [hep-ex/0507073].

[8] J. H. Lee et al. [BRAHMS Collaboration], AIP Conf. Proc. 915, 533 (2007).

[9] I. Arsene et al. [BRAHMS Collaboration], Phys. Rev. Lett. 101, 042001 (2008) [arXiv:0801.1078 [nucl-ex]].

[10] L. Adamczyk et al. [STAR Collaboration], Phys. Rev. D 86, 032006 (2012) [arXiv:1205.2735 [nucl-ex]];

[11] L. Adamczyk et al. [STAR Collaboration], Phys. Rev. D 86, 051101 (2012) [arXiv:1205.6826 [nucl-ex]].

[12] L. C. Bland et al. [AnDY Collaboration], Phys. Lett. B 750, 660 (2015) [arXiv:1304.1454 [hep-ex]].

[13] A. Adare et al. [PHENIX Collaboration], Phys. Rev. D 90, 012006 (2014) [arXiv:1312.1995 [hep-ex]]; Phys. Rev. D 90, 072008 (2014) [arXiv:1406.3541 [hep-ex]].

[14] G. Bunce et al., Phys. Rev. Lett. 36, 1113 (1976).

[15] A. Airapetian et al. [HERMES Collaboration], Phys. Lett. B 728, 183 (2014) [arXiv:1310.5070 [hep-ex]].

[16] K. Allada et al. [Jefferson Lab Hall A Collaboration], Phys. Rev. C 89, 042201 (2014) [arXiv:1311.1866 [nucl-ex]].

[17] A. Airapetian et al. [HERMES Collaboration], Phys. Lett. B 682, 351 (2010) [arXiv:0907.5369 [hep-ex]].

[18] J. Katich et al., Phys. Rev. Lett. 113, 2, 022502 (2014) [arXiv:1311.0197 [nucl-ex]].

[19] A. Abdesselam et al. [Belle Collaboration], arXiv:1611.06648 [hep-ex].

[20] C. Adolph et al. [COMPASS Collaboration], Phys. Lett. B 744, 250 (2015) [arXiv:1408.4405 [hep-ex]].

[21] A. Airapetian et al. [HERMES Collaboration], Phys. Rev. Lett. 103, 152002 (2009) [arXiv:0906.3918 [hep-ex]].

[22] A. Airapetian et al. [HERMES Collaboration], Phys. Lett. B 693, 11 (2010) [arXiv:1006.4221 [hep-ex]]. 
[23] X. Qian et al. [Jefferson Lab Hall A Collaboration], Phys. Rev. Lett. 107, 072003 (2011) [arXiv:1106.0363 [nucl-ex]].

[24] Y. X. Zhao et al. [Jefferson Lab Hall A Collaboration], Phys. Rev. C 90, 5, 055201 (2014) [arXiv:1404.7204 [nucl-ex]].

[25] L. Adamczyk et al. [STAR Collaboration], Phys. Rev. Lett. 116, 13, 132301 (2016) [arXiv:1511.06003 [nucl-ex]].

[26] M. Aghasyan et al. [COMPASS Collaboration], Phys. Rev. Lett. 119, 11, 112002 (2017) [arXiv: 1704.00488 [hep-ex]].

[27] R. Seidl et al. [Belle Collaboration], Phys. Rev. D 78, 032011 (2008) Erratum: [Phys. Rev. D 86, 039905 (2012)] [arXiv:0805.2975 [hep-ex]].

[28] J. P. Lees et al. [BaBar Collaboration], Phys. Rev. D 90, 5, 052003 (2014) [arXiv:1309.5278 [hep-ex]].

[29] J. P. Lees et al. [BaBar Collaboration], Phys. Rev. D 92, 11, 111101 (2015) [arXiv:1506.05864 [hep-ex]].

[30] M. Ablikim et al. [BESIII Collaboration], Phys. Rev. Lett. 116, 4, 042001 (2016) [arXiv:1507.06824 [hep-ex]].

[31] A. V. Efremov and O. V. Teryaev, Sov. J. Nucl. Phys. 36, 140 (1982) [Yad. Fiz. 36, 242 (1982)]; Phys. Lett. B 150, 383 (1985).

[32] J.-w. Qiu and G. F. Sterman, Phys. Rev. Lett. 67, 2264 (1991);

[33] J.-w. Qiu and G. F. Sterman, Nucl. Phys. B 378, 52 (1992);

[34] J.-w. Qiu and G. F. Sterman, Phys. Rev. D 59, 014004 (1999) [hep-ph/9806356].

[35] Y. Kanazawa and Y. Koike, Phys. Lett. B 478, 121 (2000) [hep-ph/0001021]; Phys. Lett. B 490, 99 (2000) [hep-ph/0007272].

[36] C. Kouvaris, J. W. Qiu, W. Vogelsang and F. Yuan, Phys. Rev. D 74, 114013 (2006) [arXiv:hep-ph/0609238].

[37] H. Eguchi, Y. Koike and K. Tanaka, Nucl. Phys. B 752, (2006) [arXiv:hep-ph/0604003]; Nucl. Phys. B 763, 198 (2007) [arXiv:hep-ph/0610314].

[38] Y. Koike and K. Tanaka, Phys. Lett. B 646, 232 (2007) [Erratum-ibid. B 668, 458 (2008)] [hep-ph/0612117].

[39] Y. Koike and T. Tomita, Phys. Lett. B 675, 181 (2009) [arXiv:0903.1923 [hep-ph]].

[40] H. Beppu, Y. Koike, K. Tanaka and S. Yoshida, Phys. Rev. D 82, 054005 (2010) [arXiv:1007.2034 [hep-ph]].

[41] A. Metz and D. Pitonyak, Phys. Lett. B 723, 365 (2013) [arXiv:1212.5037 [hep-ph]].

[42] K. Kanazawa and Y. Koike, Phys. Rev. D 88, 074022 (2013) [arXiv:1309.1215 [hep-ph]].

[43] H. Beppu, K. Kanazawa, Y. Koike and S. Yoshida, Phys. Rev. D 89, 034029 (2014) [arXiv:1312.6862 [hep-ph]].

[44] M. Anselmino, M. Boglione, U. D’Alesio, A. Kotzinian, F. Murgia and A. Prokudin, Phys. Rev. D 72, 094007 (2005) Erratum: [Phys. Rev. D 72, 099903 (2005)] doi:10.1103/PhysRevD.72.094007, 10.1103/PhysRevD.72.099903 [hep-ph/0507181]. 
[45] M. Anselmino, M. Boglione, U. D’Alesio, A. Kotzinian, S. Melis, F. Murgia, A. Prokudin and C. Turk, Eur. Phys. J. A 39, 89 (2009) [arXiv:0805.2677 [hep-ph]].

[46] M. Anselmino, M. Boglione, U. D’ Alesio, A. Kotzinian, F. Murgia, A. Prokudin and S. Melis, Nucl. Phys. Proc. Suppl. 191, 98 (2009) [arXiv:0812.4366 [hep-ph]].

[47] M. Anselmino, M. Boglione, U. D’Alesio, S. Melis, F. Murgia and A. Prokudin, Phys. Rev. D 87, 094019 (2013) [arXiv:1303.3822 [hep-ph]].

[48] M. Anselmino, M. Boglione, U. D’Alesio, J. O. Gonzalez Hernandez, S. Melis, F. Murgia and A. Prokudin, Phys. Rev. D 93, 3, 034025 (2016) [arXiv:1512.02252 [hep-ph]].

[49] M. Anselmino, M. Boglione, U. D’ Alesio, F. Murgia and A. Prokudin, JHEP 1704, 046 (2017) [arXiv:1612.06413 [hep-ph]].

[50] J. C. Collins and D. E. Soper, Nucl. Phys. B 193, 381 (1981) Erratum: [Nucl. Phys. B 213, 545 (1983)].

[51] J. C. Collins, D. E. Soper and G. F. Sterman, Nucl. Phys. B 250, 199 (1985).

[52] J. Collins, Foundations of Perturbative QCD, Cambridge University Press (2011).

[53] S. M. Aybat, J. C. Collins, J. W. Qiu and T. C. Rogers, Phys. Rev. D 85, 034043 (2012) doi:10.1103/PhysRevD.85.034043 [arXiv:1110.6428 [hep-ph]].

[54] M. G. Echevarria, A. Idilbi, Z. B. Kang and I. Vitev, Phys. Rev. D 89, 074013 (2014) [arXiv:1401.5078 [hep-ph]].

[55] Z. B. Kang, A. Prokudin, P. Sun and F. Yuan, Phys. Rev. D 93, 1, 014009 (2016) [arXiv:1505.05589 [hep-ph]].

[56] D. Pitonyak, Int. J. Mod. Phys. A 31, 32, 1630049 (2016) [arXiv:1608.05353 [hep-ph]].

[57] D. W. Sivers, Phys. Rev. D 41, 83 (1990).

[58] D. Boer, P. J. Mulders and F. Pijlman, Nucl. Phys. B 667, 201 (2003) [hep-ph/0303034].

[59] L. Gamberg, Z. B. Kang and A. Prokudin, Phys. Rev. Lett. 110, 23, 232301 (2013) doi:10.1103/PhysRevLett.110.232301 [arXiv:1302.3218 [hep-ph]].

[60] K. Kanazawa, Y. Koike, A. Metz and D. Pitonyak, Phys. Rev. D 91, 1, 014013 (2015) doi:10.1103/PhysRevD.91.014013 [arXiv:1410.3448 [hep-ph]].

[61] K. Kanazawa and Y. Koike, Phys. Rev. D 82, 034009 (2010) doi:10.1103/PhysRevD.82.034009 [arXiv:1005.1468 [hep-ph]].

[62] Z. B. Kang, J. W. Qiu, W. Vogelsang and F. Yuan, Phys. Rev. D 83, 094001 (2011) [arXiv:1103.1591 [hep-ph]].

[63] Z. B. Kang and A. Prokudin, Phys. Rev. D 85, 074008 (2012) [arXiv:1201.5427 [hep-ph]].

[64] A. Metz, D. Pitonyak, A. Schafer, M. Schlegel, W. Vogelsang and J. Zhou, Phys. Rev. D 86, 094039 (2012) [arXiv:1209.3138 [hep-ph]].

[65] K. Kanazawa, Y. Koike, A. Metz and D. Pitonyak, Phys. Rev. D 89, 111501 (2014) [arXiv:1404.1033 [hep-ph]].

[66] L. Gamberg, Z. B. Kang, D. Pitonyak and A. Prokudin, Phys. Lett. B 770, 242 (2017) [arXiv:1701.09170 [hep-ph]]. 
[67] K. Kanazawa, Y. Koike, A. Metz, D. Pitonyak and M. Schlegel, Phys. Rev. D 93, no. 5, 054024 (2016) doi:10.1103/PhysRevD.93.054024 [arXiv:1512.07233 [hep-ph]].

[68] D. Boer, R. Jakob and P. J. Mulders, Nucl. Phys. B 504, 345 (1997) [hep-ph/9702281].

[69] A. Bacchetta, M. Diehl, K. Goeke, A. Metz, P. J. Mulders and M. Schlegel, JHEP 0702, 093 (2007) [hep-ph/0611265].

[70] D. Boer, L. Gamberg, B. Musch and A. Prokudin, JHEP 1110, 021 (2011) [arXiv:1107.5294 [hep-ph]].

[71] M. Burkardt, Phys. Rev. D 69, 057501 (2004) [hep-ph/0311013].

[72] J. Collins, L. Gamberg, A. Prokudin, T. C. Rogers, N. Sato and B. Wang, Phys. Rev. D 94, 034014 (2016) [arXiv:1605.00671 [hep-ph]].

[73] L. Gamberg, A. Metz, D. Pitonyak and A. Prokudin, in preparation. 